\title{
AÇÃO DO ÓLEO DE NIM FRENTE À TELEÓGINAS DE RHIPICEPHALUS (BOOPHILUS) MICROPLUS EM TESTES IN VITRO
}

\author{
Andréia Buzatti ${ }^{1}$, Lew Kan Sprenger ${ }^{1}$, Thais Kucharsky ${ }^{1}$, Marcelo Beltrão Molento' \\ 1 UFPR \\ Correspondência: Andréia Buzatti: deiabuzatti@gmail.com
}

\begin{abstract}
RESUMO: O objetivo deste estudo foi avaliar a ação acaricida in vitro de um extrato a base de óleo de nim (Azaradiractha indica) comercial frente à teleóginas de Rhipicephalus (Boophilus) microplus, por meio do teste de biocarrapaticidograma/imersão. Fêmeas ingurgitadas foram coletadas de bovinos do Centro Paranaense de Referência em Agroecologia (CPRA), município de Pinhais, Paraná, Brasil. O delineamento experimental foi inteiramente casualizado com oito tratamentos e três repetições cada, consistindo em sete concentrações de óleo de nim: 0,16;0,32; 0,64; 1,28; 2,56; 5,12 e $10,24 \%$ e um grupo controle, composto por água destilada. Os resultados de eclodibilidade larval de todos os tratamentos foram semelhantes entre si, não apresentando significância estatística $(P>0,05)$. Os valores de eficiência reprodutiva $(E R)$ e de eficácia do produto (EP) foram estatisticamente significantes $(P<0,05)$. Os dados de EP variaram de $14,77 \pm 3,55$ a $40,09 \pm 2,14 \%$, correspondendo às concentrações de $0,16 \%$ e $10,24 \%$ de óleo de nim, respectivamente. Nas concentrações utilizadas o óleo de nim não interferiu no percentual de eclodibilidade larval, mas demonstrou ação na ER de $R$. (B.) microplus e na EP. O tratamento composto por $10,24 \%$ de óleo de nim foi estatisticamente superior aos demais $(P<0,05)$, apresentando $40,09 \%$ de eficácia. Os resultados demonstraram que o óleo de semente de nim apresenta potencial acaricida sendo o percentual de eficácia de cada tratamento diretamente relacionado com respectiva concentração utilizada.
\end{abstract}

Palavras-chave: Azaradiractha indica; bovinos; fitoterápicos; resistência parasitária

\section{ACTION OF NEEM OIL AGAINST THE ENGORGED FEMALES OF RHIPICEPHALUS (BOOPHILUS) MICROPLUS IN VITRO TESTS}

\begin{abstract}
The aim of this study was to evaluate the in vitro acaricide action with the tickimmersion test using neem oil (Azaradiractha indica) against the engorged females of Rhipicephalus (Boophilus) microplus. Engorged ticks were collected from cattle at the Agroecology Reference Center of Parana (CPRA), city of Pinhais, Paraná, Brazil. The design was completely randomized with eight treatments and three replicates each consisting of seven concentrations of neem oil: 0.16 , $0.32,0.64,1.28,2.56,5.12$ and $10.24 \%$ and a control group, composed of distilled water. Results from larval hatchability were similar to each other $(P>0.05)$. Reproductive efficiency and efficacy of the treatments was statistically significant $(P<0,05)$. The efficacy ranged from $14.77 \pm 3.55$ to $40.09 \pm 2.14 \%$, corresponding to concentrations of $0.16 \%$ and $10.24 \%$ neem oil, respectively. At the concentrations used neem oil did not affect the percentage of larval hatchability, but demonstrated action on reproductive efficiency of $R$. (B.) microplus and efficacy of treatments. The treatment compost of $10.24 \%$ of neem oil was statistically higher than to the others $(P<0.05)$. The results showed that neem seed oil has potential acaricide, and the percentage of efficacy of each treatment is directly related to their concentration.
\end{abstract}

Key Words: acaricide; Azaradiractha indica; cattle; phytotherapeutic 


\section{INTRODUÇÃO}

$\begin{array}{cc}\text { Rhipicephalus } & \begin{array}{r}\text { (Boophilus) } \\ \text { microplus étoparasita }\end{array} \\ \text { hematófago originário da Ásia cujo }\end{array}$ principal hospedeiro é o bovino. Possuem distribuição cosmopolita, sendo encontrados principalmente nas regiões tropicias e subtropicias (Estrada-Peña et al., 2006). Estima-se que, anualmente na América do Sul as perdas decorrentes do parasitismo ultrapassem os US $\$ 2$ bilhões (Grisi et al., 2002). No Brasil, é o ectoparasita responsável pelas maiores perdas econômicas na produção pecuária, levando a redução na produtividade, anemia, toxicidade, danos físicos e transmissão de agentes patogênicos (Gazim et al., 2011).

O controle desse ectoparasita é realizado, basicamente por meio de acaricidas químicos e a utilização desses produtos, na maioria das vezes, é feita de maneira indevida, caracterizando-se pela utilização de dosagens inadequadas, curto intervalo de aplicação, rotação incorreta dos princípios farmacológicos, acelerando o processo de resistência parasitária (RP). A RP é resultado de um processo artificial de seleção em parasitas que são capazes de sobreviver ao tratamento após o uso constante de um determinado composto (GRAF et. at., 2004). Além de acarretar a RP, essa utilização massiva aumenta o custo de produção e acarreta em acúmulo de resíduos no meio ambiente e nos produtos de origem animal o que pode ser prejudicial à saúde humana (Molento, 2005). Desde a década de 90, com o surgimento desses obstáculos à produção, métodos alternativos de controle parasitário estão sendo amplamente estudados, entre eles a utilização de produtos fitoterápicos (Martinez-Velazquez et al., 2011; Buzatti et al., 2011).
O nim, Azadirachta indica (Meliaceae), planta nativa da Índia, possui valor comercial devido à presença de princípios ativos, encontrados principalmente em suas sementes, folhas, frutos e raízes (Singh et al. 1999), que apresentam ação frente a mais de 200 espécies de organismos, como ácaros, carrapatos, nematóides, fungos e bactérias (Souza, 2002).

$O$ fruto do vegetal possui aproximadamente $40 \%$ de óleo de azadirachtina, que é o princípio ativo em maior concentração (Abdel-Shafy et al., 2002). Este metabólito interfere nos parâmetros reprodutivos dos ácaros, inibindo a biossíntese do hormônio protoracicotrópico (PTTH) e, como consequência, não ocorre a biossíntese de outros hormônios, o que impossibilita os passos normais da troca de tegumento (ecdise) e também inibe a maturação dos ovos (Aguiar-Menezes, 2005). Somado a este, observa-se atividade sinérgica de diversas substâncias, como azadirachtina, nimbina, salannina, nimbidina, kaempferol, thionemone, quercetina e outras (Bevilacqua et al., 2008). Martinez (2002) considerou que o nim tem grande importância no controle de pragas, possui largo espectro de ação, pode ser associado a outras formas de manejo, é atóxico ao homem e não prejudica o meio ambiente.

Este estudo teve por objetivo avaliar a atividade acaricida in vitro do óleo de nim frente à fêmeas ingurgitadas de $R$. (B.) microplus.

\section{MATERIAL E MÉTODOS}

Coleta dos ácaros - Fêmeas ingurgitadas de $R$. (B.) microplus foram coletadas de bovinos da raça Jersey naturalmente infestados, pertencente ao rebanho de bovinocultura de leite do Centro Paranaense de Agroecologia, CPRA, localizado no município de 
Pinhais, PR. No local, os animais não receberam qualquer antiparasitário alopático, sendo realizada somente a remoção mecânica dos carrapatos. Após a coleta, os ácaros foram acondicionados em caixas térmicas de poliestireno contendo gelo reciclável e transportados até o Laboratório de Doenças Parasitárias da Universidade Federal do Paraná, onde se procedeu com os testes in vitro.

Ensaio in vitro - $\mathrm{O}$ teste foi realizado conforme Drummond et al. (1973). Os carrapatos foram selecionados com base na motilidade normal e aparência com o corpo íntegro e máximo ingurgitamento (Leite et al., 1995), lavados em água corrente, secos em papel absorvente e pesados em grupo. O delineamento experimental foi inteiramente casualizado com oito tratamentos e três repetições cada, considerando-se 10 fêmeas ingurgitadas como cada repetição. O grupo controle (C) foi composto por água destilada:dimetilsulfóxido (DMSO) a $1 \%$ e demais grupos foram compostos por óleo de nim diluídos em água destilada:DMSO 1\%. Sendo T1: 0,16 ; T2: 0,32; T3: 0,64; T4: 1,28; T5: 2,56; T6: 5,12 e T7: $10,24 \%$ de óleo de sementes de nim de uma marca comercial, adquirido no comércio local.

Os grupos foram submetidos aos tratamentos em imersão por 5 minutos, com agitação a cada minuto. Posteriormente, as teleóginas foram retiradas da solução, secas em papel absorvente e fixadas em placas de Petri e acondicionados sob condições de $27^{\circ} \mathrm{C}$ e $80 \%$ UR durante 14 dias para realização da ovoposição. Após esta etapa, a postura total de cada grupo foi pesada, sendo armazenados $0,3 \mathrm{~g}$ de cada grupo em tubos tipo Falcon, identificados individualmente e vedados com algodão hidrófilo. Os ovos permaneceram aí por mais 26 dias, para avaliação da eclodibilidade dos ovos. A leitura foi realizada por verificação visual em intervalos de $5 \%$. Como critério, adotou-se a comparação do número de ovos remanescentes não-eclodidos com as cascas e a visualização das larvas. A eficácia dos tratamentos foi determinada de acordo com o cálculo da reprodução estimada:

$E R=\underline{\text { Peso da massa de ovos } \times \text { \%eclosão } \times 20.000^{*}}$ Peso da massa de fêmeas

$\mathrm{ER}=$ Eficiência Reprodutiva

${ }^{*}$ Constante que indica o número de ovos em $1 \mathrm{~g}$ de postura

$E P=E R$ do grupo controle - ER do grupo tratado $\times 100$ ER do grupo controle

$\mathrm{EP}=$ Eficiência do produto

$\begin{array}{ccc}\text { Análise estatística } & \text { Os dados } \\ \text { obtidos } & \text { foram } & \text { analisados }\end{array}$
individualmente, conforme cada repetição de cada tratamento, foi calculado a média da ER e a média e o desvio padrão (DP) da eclodibilidade larval e da EP. A análise estatítica foi realizada com o software GraphPad Prism 5.01. As médias de eclodibilidade larval, ER e EP foram submetidos a análise de variância, ANOVA. Resultados estatisticamente significantes $(P<0,05)$ foram submetidos ao teste de Tukey a $5 \%$ de probabilidade.

\section{RESULTADOS E DISCUSSÃO}

A eclodibilidade larval variou de $85 \pm 13,23$ a $96,67 \pm 2,89$ (Tabela 1). A análise de variância demonstrou que as diferentes concentrações de óleo de nim, composto por óleo de semente da planta, assim como o controle, composto por água destilada, não interferiram nos índices de eclodibilidade dos ácaros, proporcionando percentual de eclosão larval semelhantes entre si $(P>0,05)$. A eficiência reprodutiva $(E R)$ variou de 863660,08 a 607103,83 (Tabela 1), apresentando significância estatística $(\mathrm{P}<0,05)$. Giglioti et al. (2011), utilizaram nim, contendo concentrações 
conhecidas de azadirachtina, concluíram que o principal efeito tóxico deste princípio ativo é a interferência nos parâmetros reprodutivos do carrapato.

\begin{tabular}{|c|c|c|c|}
\hline $\begin{array}{l}\text { Concentração de } \\
\text { óleo de nim }(\%)\end{array}$ & $\begin{array}{c}\text { Eclodibilidade (\%) } \\
\text { (Média } \pm \text { DP) }\end{array}$ & $\begin{array}{c}\text { Eficiência } \\
\text { Reprodutiva(Média) }\end{array}$ & $\begin{array}{l}\text { Eficácia (\%) } \\
\text { (Média } \pm D P)\end{array}$ \\
\hline C-Controle & $96,67 \pm 2,89$ & - & $0^{\alpha}$ \\
\hline $\mathrm{T} 1-0,16$ & $96,67 \pm 5,77$ & $863660,08^{c}$ & $14,77 \pm 3,55^{\circ 0}$ \\
\hline T2- 0,32 & $93,33 \pm 5,77$ & $846721,31^{\text {be }}$ & $16,45 \pm 2,31^{\circ}$ \\
\hline T3- 0,64 & $90 \pm 10$ & $818743,17^{\circ \mathrm{cc}}$ & $19,21 \pm 11,36^{b}$ \\
\hline T4-1,28 & $91.67 \pm 7,64$ & $785737,70^{\circ \mathrm{cc}}$ & $22,46 \pm 3,42^{\circ}$ \\
\hline$T 5-2,56$ & $95 \pm 5$ & $791530,05^{\circ \mathrm{c}}$ & $21,89 \pm 5,33^{\circ}$ \\
\hline T6- 5,12 & $85 \pm 13,23$ & $711584,70^{30}$ & $29,78 \pm 3,33^{80}$ \\
\hline$T 7-10,24$ & $86,67 \pm 5,77$ & $607103,83^{\circ}$ & $40,09 \pm 2,14^{\circ}$ \\
\hline
\end{tabular}

Broglio-Micheletti et al. (2009), avaliaram a ação de extratos alcoólico e hexânico, ambos a $2 \%$, e 2 óleos emulsionáveis comerciais de Azadirachta indica A. Juss (Meliaceae). Estes autores observaram percentuais de eclodibilidade semelhantes entre grupo controle, extrato alcoólico e óleos emulsionáveis $(P>0,05)$, semelhantes aos observados no presente estudo. Apenas o extrato hexânico demonstrou ação superior sobre a eclodibilidade, sendo estatisticamente superiores aos demais tratamentos $(P<0,05)$. Silva et al.(2007) avaliaram a atividade de extratos alcoólicos de nim e capim santo sobre parâmetros reprodutivos de $R$. (B.) microplus in vitro, obtendo resultados de eclodibilidade e ER estatísticamente diferentes $(P<0,05)$, sendo o nim superior ao capim santo e ao grupo controle. Srivastava et al. (2008), utilizaram extrato bruto seco de nim em sete concentrações, variando de 2 a $8 \%$, demonstrando interferência na ER das teleóginas, sendo a concentração $8 \%$ estatísticamente superior as demais $(P<0,05)$. Nesta pesquisa o tratamento composto por $10,24 \%$ de óleo de nim foi estatisticamente superior aos demais $(P<0,05)$.

Estudos com delineamento experimental semelhante, mas utilizando compostos vegetais de origem diferente podem proporcionar resultados discrepantes. Isto pode ocorrer devido à falta de informação sobre a composição química dos extratos e óleos utilizados (Martins-Ramos, et al. 2010). O teor de compostos químicos é variável, a síntese destas substâncias ainda que orientada pelas características genéticas da planta, é controlada por fatores do ecossistema como iluminação, temperatura, composição do solo e umidade (Lapa, 2004). Segundo Heimerdinger et al., (2006), além das variações nas condições edafoclimáticas, a forma de cultivo e a conservação dos materiais vegetais podem implicar em oscilações em sua composição química. Yakkundi et al. (1995) avaliaram o teor de azadiractina em sementes de A.indica, observando redução de 5 e 35\% após um e quatro meses de armazenamento, respectivamente. Da mesma forma, Johnson et al. (1996) observaram decréscimo nos teores de azadiractina e salannina após seis meses de armazenamento.

Os dados de EP apresentaram significância estatística $(P<0,05)$, variando de $14,77 \pm 3,55$ a $40,09 \pm 2,14 \%$, correspondente às concentrações de $0,16 \%$ e $10,24 \%$ de óleo de nim, respectivamente (Tabela 1). BroglioMicheletti et al. (2009), avaliaram a eficácia de extratos alcoólico e hexânico, ambos a 2\%, e 2 óleos emulsionáveis comerciais de nim, observando resultados superiores aos deste estudo. O óleo emusionável 2 (Base Nim®) e o extrato hexânico apresentaram eficácia de 65,50\% e $73,20 \%$, respectivamente, sendo superiores aos demais tratamentos avaliados $(P<0,05)$. Estes autores usaram dimetilsulfóxido (DMSO) a $1 \%$ como solubilizante dos tratamentos e formaram um grupo controle composto por água+DMSO, o qual apresentou 
eficácia de 21,42\%, sendo superior ao óleo emulsionável 3(Nim-I-Goß) e ao extrato etanólico $(P<0,05)$. Segundo Chagas et al. (2003), DMSO é um excelente permeabilizador de membranas, permitindo a penetração do produto a ser testado de maneira rápida. No entanto, pode ser capaz de causar alta mortalidade em $R$. (B.) microplus, mesmo em baixas concentrações, prejudicando a determinação de eficácia do tratamento.

Giglioti et al. (2011), avaliaram óleo de semente de nim contendo teores conhecidos de azadirachtina, e observaram uma relação dosedependente direta na eficácia do tratamento. $O$ tratamento composto por $10 \%$ de óleo de nim e 10,000 ppm de azadirachtina demonstrou 0 maior percentual de eficácia $(94,7 \%)$. Valente et al. (2007), compararam in vivo a eficácia do extrato aquoso de folhas frescas de nim (1 kg de folhas frescas, diluída em $5 \mathrm{~L}$ de água), aplicado em banhos semanais (2L/animal) durante um mês, com o tratamento com abamectina pour on, em uma aplicação para controle de $R$. (B). microplus. Os dois tratamentos demonstraram eficácia semelhante $(P>0.0001)$, e os autores afirmaram que o extrato vegetal de nim poderia substituir a abamectina em um programa de carrapatos no semiárido nordestino.

Considerando a eficácia obtida pelo óleo de nim no presente estudo, o mesmo pode ser associado a um controle acaricida convencional, a base de medicamentos alopáticos, visando um efeito sinérgico na resposta frente ao desafio no controle de carrapatos. Os fitoterápicos devem ser usados em controles integrados parasitários, pois os mesmos muitas vezes não apresentam uma atividade muito alta; não obstante o seu uso combinado pode alavancar a eficácia na forma combinada (Carmona; Pereira, 2013). Contudo, deve-se considerar a concentração utilizada dos fármacos, pois a eficácia do referido óleo demonstrou possuir um efeito dosedependente.

\section{CONCLUSÃO}

No delineamento experimental aplicado os resultados demonstraram que 0 óleo de semente de nim apresenta potencial acaricida, sendo o percentual de eficácia de cada tratamento diretamente relacionado com respectiva concentração utilizada.

\section{REFERÊNCIAS}

ABDEL-SHAFY, S.; ZAYED, A.A. [2002]. In vitro acaricidal effect of plant extract of Neem seed oil (Azadirachta indica) on egg, immature, and adult stages of Hyalomma anatolicum excavatum (Ixodoidea:Ixodidade). Veterinary Parasitology, v.106, n.1, p.89-96, 2002.

\section{AGUIAR-MENEZES, E. L. Inseticidas}

Botânicos: seus princípios ativos, modo de ação e uso agrícola. Seropédica: Embrapa Agrobiologia, 2005. 58 p.

BEVILACQUA, A.H.V. ; Suffredini, I.B.; Bernardi, M.M. [2008] Toxicidade de Neem, Azadirachta indica A. Juss. (Meliaceae), em Artemia sp: comparação da preparação comercial e do óleo puro. Revista do Instituto de Ciências da Saúde, v.26, n.2, p. 57-160, 2008.

BROGLIO-MICHELETTI, S.M.F.; VALENTE, E.C.N.; SOUZA, L. A. de; et al. [2009]. Extratos de plantas no controle de Rhipicephalus (Boophilus) microplus (Canestrini, 1887) (Acari: Ixodidae) em laboratório. Revista Brasileira de Parasitologia Veterinária, v.18, n.4, p.44-48, 2009.

BUZATTI, A.; KRAWCZAK, F. S.; PIVOTO, F. $\mathrm{L}$.; et al. Atividade acaricida in vitro de Glechon spathulata Benth. sobre teleóginas de Rhipicephalus (Boophilus) microplus. Ciência Rural, v.41, n.10, p.1813-1817, 2011.

CARMONA, F.; PEREIRA, A. M. S. Herbal medicines: old and new concepts, truths and misunderstandings. Revista Brasileira de Farmacognosia, v.23, n.2, p.379-385, 2013.

CHAGAS, A.C.S.; LEITE, R.C.; FURLONG, J.; et al. [2003]. Sensibilidade do carrapato Boophilus microplus a solventes. Ciência Rural, v.33, n.1, p.109-114, 2003. 
DRUMOND, R.O.; ERNST, S.E.; TREVINO, J.L.; et al. [1973]Boophilus annulatus and Boophilus microplus: Laboratory tests of insecticides.

Journal Economic Entomology, v.66, n.1, p.130-133, 1973.

ESTRADA-PEÑA, A.; BOUATTOUR, A.; CAMICAS, J.L.; et al. [2006]. The known distribution and ecological preferences of the tick subgenus Boophilus (Acarilxodidae) in Africa and Latin America . Experimental and

Applied Acarology, v.38, n.1, p.219-235, 2006.

GAZIM, Z.C ; DEMARCHI, I.G.; LONARDONI, M.V.; et al. [2011]Acaricidal activity of the essential oil from Tetradenia riparia (Lamiaceae) on the cattle tick Rhipicephalus (Boophilus) microplus (Acari; Ixodidae). Experimental Parasitology, v.129, n.2, p.175-178, 2011.

GIGLIOTI, R.; FORIM, M.R.; OLIVEIRA, H.N.; et al. [2011] In vitro acaricidal activity of neem (Azadirachta indica) seed extracts with known azadirachtin concentrations against Rhipicephalus microplus. Veterinary

Parasitology, v.181, n.2/4, p.309-315, 2011.

GRAF, J.F.; GOGOLEWSKI, R.; LEACH-BING, N.; et al. Tick control: an industry point of view Parasitology, v.129, n.1, p.427-442, 2004.

GRISI, L.; MASSARD, C.L.; MOYA BORJA, G.E.; et al. [2002]. Impacto econômico das principais ectoparasitoses em bovinos no Brasil. A Hora Veterinária, v.21, n.125, p.8-10, 2002.

HEIMERDINGER, A.; OLIVO, C.J.; MOLENTO, M.B.; et al. [2006]. Alcoholic extract of lemongrass (Cymbopogon citratus) on the control of Boophilus microplus in cattle. Rev.

Bras. de Parasit. Vet., v.15, n.1, p.37-39, 2006.

JOHNSON, S.; MORGAN, E.D.; PEIRIS, C.N.; et al. [1996]. Development of the major triterpenoids and oil in the fruit and seeds of neem (Azadirachta indica). Annals of Botany, v.78, n.3, p.383-388, 1996.

LAPA, A.J.; SOUCCAR, C.; LIMA-LANDMAN, M.T.R.; et al. Farmacologia e toxicologia de produtos naturais. In SIMÕES, C.M.O. SCHENKEL, E.P.; GOSMAN G.; et al.

Farmacognosia: da planta ao medicamento. Porto Alegre: Ed. Universidade, 2004. UFRGS, p. 247-262. 5.ed.

LEITE, R.C.; LABRUNA, M.B.; OLIVEIRA, P.R.; et al. [1995]. In vitro susceptibility of engorged females from different populations of Boophilus microplus to commercial acaricides. Revista Brasileira de Parasitologia Veterinária, v.4, n.2, p.283-294, 1995.
MARTINEZ, S.S. O Nim. Natureza, usos múltiplos, produção. Londrina: IAPAR, 2002. $142 \mathrm{p}$.

MARTINS-RAMOS, D.; BORTOLUZZI, R.L.C.; MANTOVANI, A.; et al. [2010]. Plantas medicinais de um remascente de Floresta Ombrófila Mista Altomontana, Urupema, Santa Catarina, Brasil. Revista Brasileira de Plantas Medicinais, v.12, n.3, p.380-397, 2010.

MARTINEZ-VELAZQUEZ, M.; CASTILLOHERRERA, G.A.; ROSARIO-CRUZ, R.; et al. [2011]. Acaricidal effect and chemical composition of essential oils extracted from Cuminum cyminum, Pimenta dioica and Ocimum basilicum against the cattle tick Rhipicephalus (Boophilus) microplus (Acari: Ixodidae).

Parasitology Res., v.100, n.2, p.481-487, 2011.

MOLENTO, M. [2005]. Resistência parasitaria em helmintos de eqüídeos e propostas de manejo. Ciência Rural, v.35, n.6, p.1469-1477, 2005.

SILVA, W.W. ; ATHAYDE, A.C.R.;

RODRIGUES, O.G.; et al. [2007]. Efeitos do neem (Azadirachta indica A. Juss) e do capim santo [Cymbopogon citratus (DC) Stapf] sobre os parâmetros reprodutivos de fêmeas ingurgitadas de Boophilus microplus e Rhipicephalus sanguineus (Acari: Ixodidae) no semiárido paraibano. Revista Brasileira de Plantas Medicinais, v.9, n.3, p.1-5, 2007.

SINGH, A.; NEGI, M. S.; RAJAGOPAL, J.; et al. Assessment of genetic diversity in Azadirachta indica using AFLP markers. Theoretical and Applied Genetics, v.99, n.1-2, p.272-279,1999.

SOUZA, E.C.P.M. Métodos em ecotoxicologia marinha: aplicações no Brasil. In:

Métodos de ecotoxicologia marinha. São Paulo: Artes Gráficas, 2002. p.9-14.

SRIVASTAVA, R.; S. GHOSH; D. B. MANDAL; et al. [2008]. Efficacy of Azadirachta indica extracts against Boophilus microplus. Parasitology Research, v.104, n.1, p.149-153, 2008.

VALENTE, M.; BARRANCO, A.; SELLAIVEVILLAROEL, A.B.; et al. [2007]. Eficácia do extrato aquoso de Azadiracta indica no controle de Boophilus microplus em bovino. Arq. Bras. de Med. Vet. e Zoote., v.59, n.5, p.1341-1343, 2007.

YAKKUNDI, S.R.; THEJAVATHI, R.; RAVINDRANATH, B. [1995]. Variation of Azadirachtin content during growth and storage of neem (Azadirachta indica) seeds. Journal of Agricultural and Food Chemistry, v.43, n.9, p.2517-2519, 1995. 